\title{
Disease Activity, Physical Function, and Radiographic Progression After Longterm Therapy with Adalimumab Plus Methotrexate: 5-Year Results of PREMIER
}

\author{
DÉSIRÉE van der HEIJDE, FERDINAND C. BREEDVELD, ARTHUR KAVANAUGH, EDWARD C. KEYSTONE, \\ ROBERT LANDEWÉ, KAUSHIK PATRA, and AILEEN L. PANGAN
}

\begin{abstract}
Objective. To evaluate the efficacy and safety of initial combination treatment with adalimumab (ADA) and methotrexate (MTX) versus monotherapy with ADA or MTX during an open-label extension of PREMIER.

Methods. Patients with early rheumatoid arthritis (RA) received blinded ADA plus MTX, ADA alone, or MTX alone for 2 years in PREMIER. At Year 2, patients could enroll in an open-label extension and receive ADA monotherapy; MTX could be added at the investigator's discretion. Longterm efficacy results are presented as observed data.

Results. In the open-label period, 497 of the original 799 randomized patients had $\geq 1$ dose of ADA (by original randomization: ADA plus MTX, $\mathrm{n}=183$; ADA, $\mathrm{n}=159$; MTX, $\mathrm{n}=155$ ). In the completers cohort [patients with available Year-5 ACR responses and modified total Sharp scores (mTSS)], the Year-5 mean change from baseline in mTSS for the ADA+MTX arm $(\mathrm{n}=124)$ was 2.9, compared with 8.7 and 9.7 in the ADA $(n=115)$ and MTX $(n=115)$ arms. Comprehensive disease remission, defined as the combination of DAS28 remission, normal function (Health Assessment Questionnaire $\leq 0.5)$, and radiographic nonprogression $(\Delta \mathrm{mTSS} \leq 0.5)$, was achieved by more patients in the initial ADA+MTX arm (35\%) than in the ADA (13\%) or MTX (14\%) arms. Conclusion. Initial combination treatment with ADA plus MTX, followed by open-label ADA, led to better longterm clinical, functional, and radiographic outcomes than either initial ADA or MTX monotherapy during 5 years of treatment. (First Release Oct 1 2010; J Rheumatol 2010;37:2237-46; doi:10.3899/jrheum.100208)
\end{abstract}

Key Indexing Terms:

ADALIMUMAB RHEUMATOID ARTHRITIS

\section{RADIOGRAPHIC PROGRESSION PHYSICAL FUNCTION} LONGTERM TREATMENT

From Leiden University Medical Center, Leiden, The Netherlands; University of California San Diego Center for Innovative Therapy, La Jolla, California, USA; University of Toronto, Toronto, Ontario, Canada; Department of Rheumatology, University of Maastricht, Maastricht, The Netherlands; and Abbott Laboratories, Abbott Park, Illinois, USA.

Supported by Abbott Laboratories, Abbott Park, IL, USA. Dr. van der Heijde has received consulting fees from Abbott, Amgen, Aventis, Bristol Myers Squibb, Centocor, Pfizer, Roche, Schering Plough, UCB, and Wyeth. Dr. Breedveld has received consulting fees from Centocor, Schering-Plough, Amgen/Wyeth, and Abbott. Dr. Kavanaugh has received research grants from Abbott. Dr. Keystone has received research grants from Abbott, Centocor, and Amgen. Dr. Landewé has received research grants from Abbott, Amgen, Centocor, Schering, UCB, and Wyeth. Dr. Patra is an Abbott employee. Dr. Pangan is an Abbott employee and holds stock in Abbott.

D. van der Heijde, MD, PhD; F.C. Breedveld, MD, PhD, Leiden University Medical Center; A. Kavanaugh, MD, University of California San Diego Center for Innovative Therapy; E.C. Keystone, MD, University of Toronto; R. Landewé, MD, Department of Rheumatology, University of Maastricht; K. Patra, PhD; A.L. Pangan, MD, Abbott Laboratories.

Address correspondence to Dr. A.L. Pangan, Immunology Development, Abbott Laboratories, 100 Abbott Park Road, Abbott Park, IL 60064-3500. E-mail: aileen.pangan@abbott.com

Full Release Article. For details see Reprints/Permissions at jrheum.org Accepted for publication June 23, 2010.
In patients with rheumatoid arthritis (RA) and aggressive disease, joint erosions may occur as early as within 6 months of disease onset. The progression of damage then occurs rapidly, especially during the first 2 years ${ }^{1,2}$. The optimal management approach for such patients takes into consideration the appropriate type and timing of therapy. It has been shown that early identification and treatment of RA can reduce disease progression and loss of function ${ }^{3}$. However, few studies have prospectively evaluated the influence of early RA treatment on prevention of both radiographic damage and clinical disease progression, especially over the long term ${ }^{4}$.

Studies have shown that a combination of a tumor necrosis factor (TNF) antagonist plus methotrexate (MTX) is superior to MTX alone in significantly improving clinical signs and symptoms of RA and inhibiting radiographic progression $^{5,6,7}$. TNF antagonist treatment has also been shown to significantly improve physical functioning and healthrelated quality of life $^{8}$, as well as employment outcomes such as job loss ${ }^{9}$, and work productivity ${ }^{10}$. 
PREMIER was a 2-year, randomized, double-blind study of patients with early RA. The design included 3 treatment arms representing 3 therapeutic options available to rheumatologists for treating patients with early RA: a combination of an anti-TNF agent plus MTX [adalimumab (ADA) plus MTX], monotherapy with an anti-TNF agent (ADA), and monotherapy with MTX. At the end of Year 2, combination treatment with ADA+MTX led to significantly better clinical responses, physical functioning, and radiographic inhibition than either ADA alone or MTX alone ${ }^{11}$.

This report presents data from patients who participated in an ongoing open-label extension study of PREMIER. Patients completed 2 years of double-blind treatment and 3 years of open-label ADA therapy, allowing an analysis of 5 total years of treatment. We sought to determine whether there was a sustained advantage of initial combination therapy over either ADA or MTX monotherapy that could be observed even after 3 years of equal opportunity for openlabel ADA therapy.

\section{MATERIALS AND METHODS}

Study design. For the first 2 years of PREMIER, the study was a multicenter, randomized, active comparator-controlled, double-blind clinical trial. The first patient was enrolled in January 2001. Patients were assigned to 1 of 3 treatments: MTX monotherapy (initiated at a dosage of $7.5 \mathrm{mg}$ weekly and increased to a maximum $20 \mathrm{mg}$ weekly, as tolerated), ADA monotherapy (40 mg subcutaneously every other week), or combination therapy with ADA+MTX. Efficacy and safety methods and results from the double-blind period have been reported ${ }^{11}$. At Year 2, all patients who were still on blinded therapy were permitted to enter the open-label phase; these patients were switched to open-label ADA monotherapy (40 mg subcutaneously every other week). Blinded MTX and placebo were discontinued, but open-label MTX (at any dosage, with a maximum of $20 \mathrm{mg}$ weekly) could be added at any time after the start of the open-label period, based on investigator's clinical judgment.

Patients. The PREMIER inclusion and exclusion criteria have been described in detail ${ }^{11}$. The main inclusion criteria were as follows: $\geq 18$ years of age; American College of Rheumatology (ACR) rating scale 1987 revised classification criteria for $\mathrm{RA}^{12}$ with disease duration $<3$ years; swollen joint count $\geq 8$, tender joint count $\geq 10$, erythrocyte sedimentation rate $\geq 28 \mathrm{~mm} / \mathrm{h}$ or C-reactive protein concentration $\geq 1.5 \mathrm{mg} / \mathrm{dl}$, and $\geq 1$ joint erosion or rheumatoid factor positivity. All patients were naive to MTX therapy at study entry.

All patients who had at least 1 dose of open-label ADA were included in analyses of concomitant MTX use and serious adverse events (SAE) during the open-label period. All patients with data available for each timepoint were included in the efficacy analyses for ACR response, Disease Activity Score 28-joint count (DAS28) remission, and Health Assessment Questionnaire (HAQ) scores over time. For radiographic analyses and the analysis of comprehensive disease remission, only patients in the 5-year completers cohort were included. The 5-year completers cohort was defined as patients with available ACR response scores and changes in modified total Sharp scores (mTSS) at Year 5.

Efficacy and safety measures. Clinical signs and symptoms were measured by $70 \%$ and $90 \%$ improvement in the ACR response criteria (ACR70 and ACR90, respectively) and DAS28 ${ }^{13}$. Patients were considered to have achieved clinical remission if they achieved DAS28 $<2.6$. Physical function was measured by the HAQ ${ }^{14}$.

To measure radiographic progression in patients who completed and had radiographs at Year 5, 2 readers who were blinded to patient and sequence evaluated each radiograph. Radiographs at baseline and Years 2, 3 , and 5 were all evaluated at the same reading session using the modified total Sharp score (mTSS; range 0-398), as described ${ }^{5}$. Changes from baseline mTSS, joint erosion, and joint space narrowing (JSN) scores were calculated at Years 2, 3, and 5.

The percentage of patients who achieved comprehensive disease remission was also determined, as measured by concurrent presence of the following triple criteria: clinical remission (DAS28 < 2.6), normal physical function (HAQ $\leq 0.5$ ), and radiographic nonprogression (change from baseline mTSS $\leq 0.5$ ).

Safety was assessed by evaluation of SAE of interest reported during the open-label period of the trial.

Statistical methods. Efficacy analyses used observed data. Descriptive analyses were performed on all efficacy variables by the originally assigned treatment group. Percentages of patients achieving ACR responses and mean changes from baseline radiographic scores are reported at all assessment points using the original study baseline values as reference points.

The potential effects of concomitant MTX use during the open-label period on efficacy were evaluated by using a generalized estimating equation (GEE) repeated-measures logistic model on ACR50 responses, DAS28 remission rates, and $\mathrm{HAQ} \leq 0.05$ at any time during the open-label period (Years 3-5). Concomitant MTX use was defined as $\geq 12$ weeks of continuous MTX use, which was considered to be sufficient time for a therapeutic trial of MTX ${ }^{15}$. Given the complex nature of MTX use in the open-label period, modeling-based averaging was done to avoid the confounding factors in an analysis that simply compares outcome variables before MTX initiation and at a fixed timepoint after start of MTX.

\section{RESULTS}

Disposition and patient flow. A total of 799 patients were enrolled in PREMIER (ADA+MTX, $\mathrm{n}=268$; ADA, $\mathrm{n}=$ 274; MTX, $n=257$ ). Of these, 497 patients had at least 1 dose of ADA in the open-label period (ADA+MTX, $\mathrm{n}=183$; ADA, $n=159$; MTX, $n=155$ ). During the open-label period, 109 patients discontinued the study. Reasons for discontinuation included the following (ADA+MTX, ADA, MTX, respectively): adverse events $(14.2 \%, 10.7 \%, 7.7 \%)$, withdrew consent $(8.2 \%, 6.9 \%, 7.7 \%)$, lack of efficacy $(3.3 \%$, $1.9 \%, 7.1 \%)$, and death $(1.1 \%, 0.6 \%, 0.6 \%)$. Patients may have had more than 1 reason for discontinuation. Of the 388 patients who completed 5 years of the study (according to original randomized treatment group: ADA+MTX, $\mathrm{n}=135$; ADA, $n=126$; MTX, $n=127), 354$ were included in the 5-year completers cohort $(\mathrm{ADA}+\mathrm{MTX}, \mathrm{n}=124 ;$ ADA, $\mathrm{n}=$ 115; MTX, $\mathrm{n}=115$ ).

Baseline characteristics. Baseline disease characteristics in the 5-year completers cohort were similar to those of the full randomized study population, except that radiographic scores were generally numerically lower in the 5 -year completers cohort (Table 1).

Use of MTX during open-label treatment. Overall, the original treatment arms had similar percentages of patients who received MTX and similar numbers of weeks of exposure to MTX during the open-label period (Figure 1). The median numbers of weeks from the start of the open-label period to the addition of MTX were 18 (ADA+MTX), 25 (ADA), and 12 (MTX) weeks. The percentages of patients in each treatment arm who received at least 12 consecutive weeks of 
Table 1. Baseline disease characteristics in the randomized and 5-year completers cohorts. Statistics are group means except where noted.

\begin{tabular}{lcccccc}
\hline & \multicolumn{3}{c}{ Randomized Cohort* } & \multicolumn{3}{c}{ 5-Year Completers Cohort by } \\
& ADA+MTX, & ADA, & MTX, & Original Randomization Arm \\
ADA+MTX, & ADA, & MTX, \\
Characteristic & $\mathrm{n}=268$ & $\mathrm{n}=274$ & $\mathrm{n}=257$ & $\mathrm{n}=124$ & $\mathrm{n}=115$ & $\mathrm{n}=115$ \\
\hline RA duration, yrs & 0.7 & 0.7 & 0.8 & 0.8 & 0.7 & 0.8 \\
SJC (66) & 21.1 & 21.8 & 22.1 & 21.1 & 21.0 & 22.9 \\
TJC (68) & 30.7 & 31.8 & 32.3 & 30.7 & 32.8 & 31.6 \\
DAS28 & 6.3 & 6.4 & 6.3 & 6.3 & 6.3 & 6.2 \\
HAQ & 1.5 & 1.6 & 1.5 & 1.4 & 1.6 & 1.5 \\
CRP, mg/dl & 3.9 & 4.1 & 4.0 & 3.9 & 3.7 & 3.7 \\
mTSS & 18.1 & 18.8 & 21.9 & 15.5 & 16.8 & 19.5 \\
JE & 11.0 & 11.3 & 13.6 & 9.4 & 9.7 & 10.7 \\
JSN & 7.1 & 7.5 & 8.2 & 6.1 & 7.1 & 8.8 \\
Estimated annual mTSS progression & & & & & & \\
\multicolumn{1}{c}{ median }
\end{tabular}

* Data from Breedveld, et al ${ }^{11}$ except for "estimated annual mTSS progression." ${ }^{\dagger}$ Median of the estimated individual patient annual mTSS progression. ADA: adalimumab; DAS28: 28-joint Disease Activity Score; HAQ: Health Assessment Questionnaire; JE: joint erosions; JSN: joint space narrowing; mTSS: modified total Sharp score; MTX: methotrexate; SJC: swollen joint count; TJC: tender joint count.

MTX during the open-label period were 42\% (ADA+MTX), $50 \%$ (ADA), and 38\% (MTX). Approximately $90 \%$ of those who had at least 12 weeks of continuous MTX use had 24 weeks of MTX use. The mean weekly dosages of MTX for these patients were similar between original treatment arms (ADA+MTX, 14.9 mg; ADA, $14.7 \mathrm{mg}$; and MTX, $14.3 \mathrm{mg}$ ).

To determine whether disease activity triggered the addition of MTX during the open-label period, we calculated the mean DAS28 score at the visit during which MTX was restarted for each of the original randomized treatment groups. The means were similar for each of the groups: ADA+MTX 3.26 (95\% CI 2.94, 3.57); ADA 3.63 (95\% CI 3.26, 4.01); and MTX 3.65 (95\% CI 3.32, 3.98).
We analyzed Year 5 data according to the original randomized treatment groups without differentiating between those who were receiving concurrent MTX therapy and those who were not during the open-label period. A GEE repeated-measures logistic regression model was employed to determine whether there was a relationship between concomitant MTX use ( $\geq 12$ weeks of continuous use) and ACR50 response at any time during Years 3 to 5, with adjustment for double-blind treatment arm. In the initial model with all 3 original treatment groups, the ADA and MTX monotherapy arms did not demonstrate any statistically significant difference. Therefore, the 2 original monotherapy groups were combined in the final model,

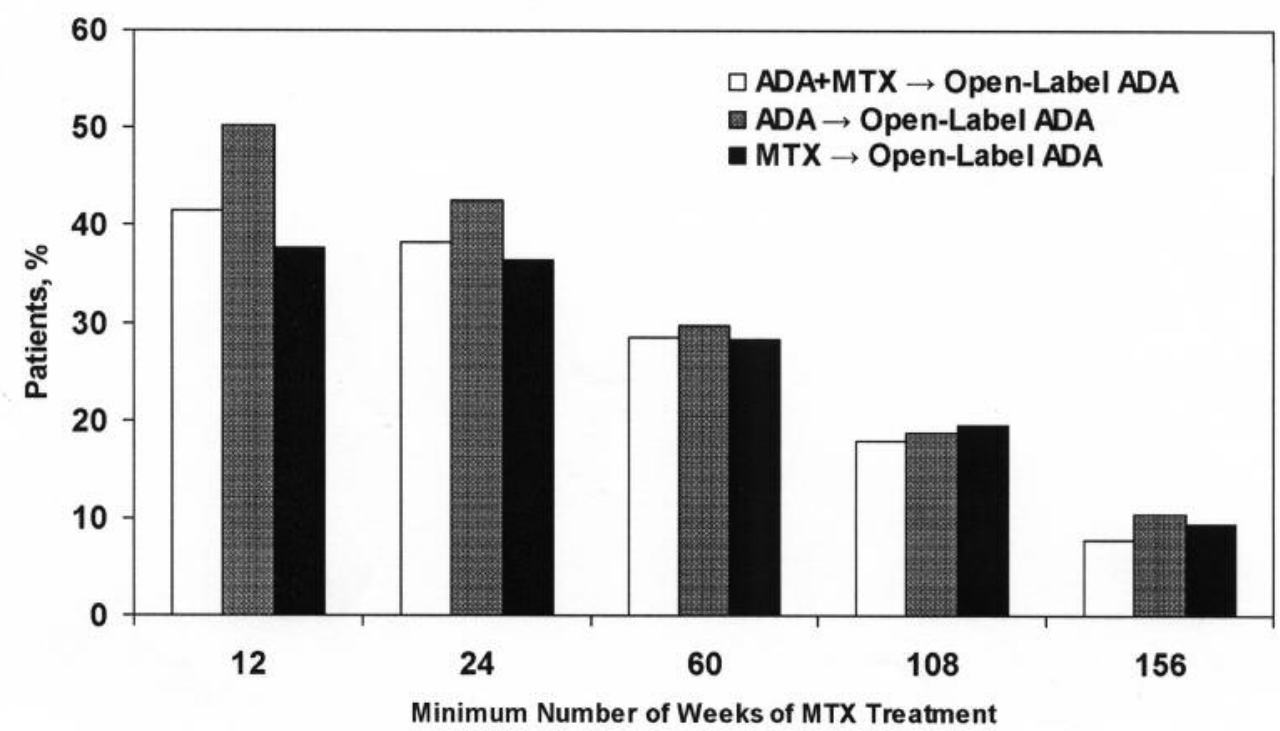

Figure 1. Patient distribution by minimum number of weeks of methotrexate (MTX) therapy received during open-label adalimumab (ADA) treatment. 
essentially for adjustment for combination treatment versus monotherapy in the open-label period. Overall, ACR50 responses by the GEE model were similar for patients with and without concomitant MTX use during the open-label period. This was true for both the randomized monotherapy groups (with MTX, 68\%; without MTX, 70\%) and the randomized ADA+MTX group (with MTX, 77\%; without MTX, 79\%). Results of a similar GEE model on DAS28 remission data were consistent with the results from the ACR50 response data for both the monotherapy group (with MTX, 50\%; without MTX, 49\%) and the ADA+MTX group (with MTX, 65\%; without MTX, 63\%). The same GEE model applied for HAQ $\leq 0.5$ resulted in a statistically significant difference for both the monotherapy group (with MTX, 52\%; without MTX, 58\%) and the ADA+MTX group (with MTX, 66\%; without MTX, 71\%). However, these results still demonstrate that the addition of MTX does not increase the likelihood of achieving HAQ $\leq 0.5$ as the percentages were lower for the group who received MTX than for those who did not.

Efficacy. Clinical response measures. Figure 2 presents the percentages of patients with DAS28 remission and ACR70 and ACR90 responses over time for each original treatment arm, based on the numbers of patients with data available at each timepoint. At Year 5, ACR70 response was achieved by $64 \%$ of the combination therapy group, $53 \%$ of the ADA group, and $57 \%$ of the MTX group. DAS28 remission at Year 5 was achieved by $61 \%$ of the combination therapy group, $52 \%$ of the ADA group, and 56\% of the MTX group. The overall pattern during the open-label period (Years 3-5) was one of superior response for patients in the initial combination therapy arm, although the differences between treatment arms decreased by Year 5 .

Physical function. Mean HAQ decreased for all 3 treatment arms during the double-blind treatment period (Figure 3), and improvements were sustained through Year 5. However, patients in the original ADA+MTX arm continued to have the lowest mean HAQ (best functional status) at each assessment point throughout both the double-blind and open-label periods.

Radiographic measures. Mean changes in mTSS, joint erosion, and JSN scores from baseline to Years 2, 3, and 5 for the 5-year completers cohort are presented in Figure 4. Better inhibition of radiographic progression occurred in the initial combination therapy group than in either monotherapy group. At Year 5, mean change from baseline mTSS score was least in the combination therapy group (2.9), compared with the ADA (8.7) and MTX (9.7) monotherapy groups. In the ADA+MTX group, $53 \%$ of patients had no radiographic progression at Year 5, compared with only $34 \%$ and $33 \%$ in the ADA monotherapy and MTX monotherapy groups, respectively. Joint erosion and JSN scores demonstrated a similar pattern of less progression in the combination therapy group than in either monotherapy group.
The patients included in the completers cohort who started on either MTX or ADA monotherapy had similar radiographic changes at Year 2 and Year 5. In the published PREMIER results from the 2-year double-blind period ${ }^{11}$, however, the MTX monotherapy group had greater progression than the ADA group on all radiographic measures at Year 2 (represented by broken lines in the left panels of each graph in Figure 4). Analysis of Year 2 radiographic scores for the subset of patients who were not included in the 5-year completers cohort (i.e., patients who had Year 2 radiographs but not Year 5 radiographs) indicated that patients in the MTX group who were not included had numerically worse progression than did patients from the ADA group who were not included [mean change in mTSS at Year 2 for ADA was $4.8(95 \%$ CI $0.3,9.3 ; \mathrm{n}=41)$ and for MTX was $7.2(95 \% \mathrm{CI}$ $3.1,11.2 ; \mathrm{n}=39)]$.

Comprehensive disease remission (triple criteria). Figure 5 presents the percentage of patients in the 5-year completers cohort with data available who achieved comprehensive disease remission according to the triple criteria [clinical remission (DAS28 < 2.6), normal function (HAQ $\leq 0.5$ ), and radiographic nonprogression (change in $\mathrm{mTSS} \leq 0.5$ )]. When DAS28 remission at Year 5 was used as the sole measure of effectiveness, this was achieved by $60 \%$ of the ADA+MTX group, $52 \%$ of the ADA group, and $57 \%$ of the MTX group in the 5-year completers cohort. The differences in efficacy results between the 3 original treatment groups were more marked when the concurrent presence of DAS28 remission and radiographic nonprogression was required. More patients in the combination therapy group (35\%) than in either monotherapy group (ADA, 13\%; MTX, 14\%) achieved comprehensive disease remission according to the triple criteria.

Safety. During the open-label period, 497 patients received at least 1 dose of ADA, representing a total of 1,335.2 patient-years (PY). Serious infections occurred at the rate of 3.3 events per $100 \mathrm{PY}$. There were 2 cases of tuberculosis (TB) reported (rate of 0.1/100 PY) - a case of disseminated TB in a 49-year-old woman from the US with a history of TB as a child and unknown treatment history who was PPD-positive and received 6 months of isoniazid prophylaxis during the study; and a case of gastrointestinal TB in a 78-year-old man from France. Both TB cases were previously randomized to the ADA+MTX arm. No SAE cases of opportunistic infections occurred. One SAE case each of lymphoma $(<0.1 / 100 \mathrm{PY})$ and nonmelanoma skin cancer $(<$ $0.1 / 100 \mathrm{PY}$ ) occurred. The rate for malignancies other than lymphomas and nonmelanoma skin cancer that were reported as SAE by the investigators was $0.8 / 100 \mathrm{PY}$. This rate represents 11 reported cases of malignancies -3 cases of breast cancer, 2 cases of bladder cancer, and 1 case each of malignant melanoma, tongue neoplasm, pancreatic neoplasm, and lung, gastric and colon cancers. There were no SAE cases of lupus-like syndrome or demyelinating disease.

Personal non-commercial use only. The Journal of Rheumatology Copyright @ 2010. All rights reserved. 
A
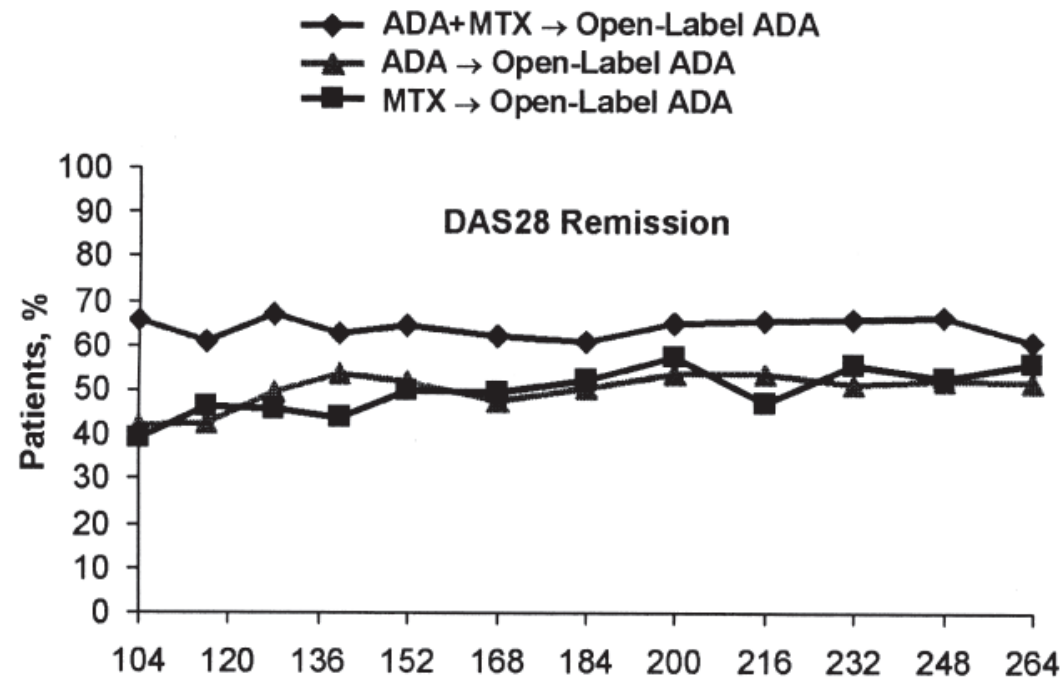

Study Week

B

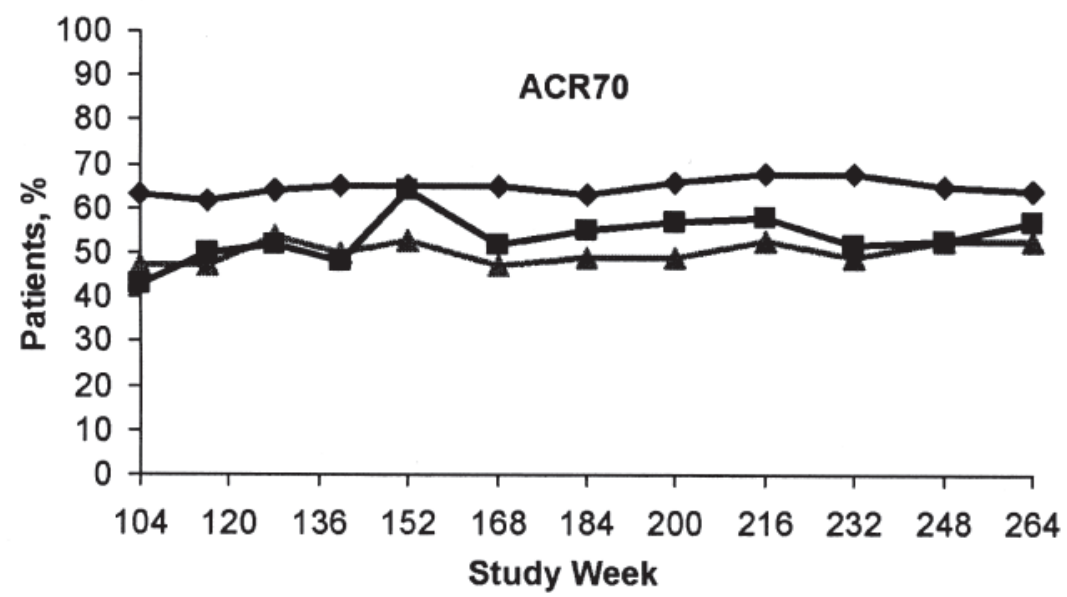

C

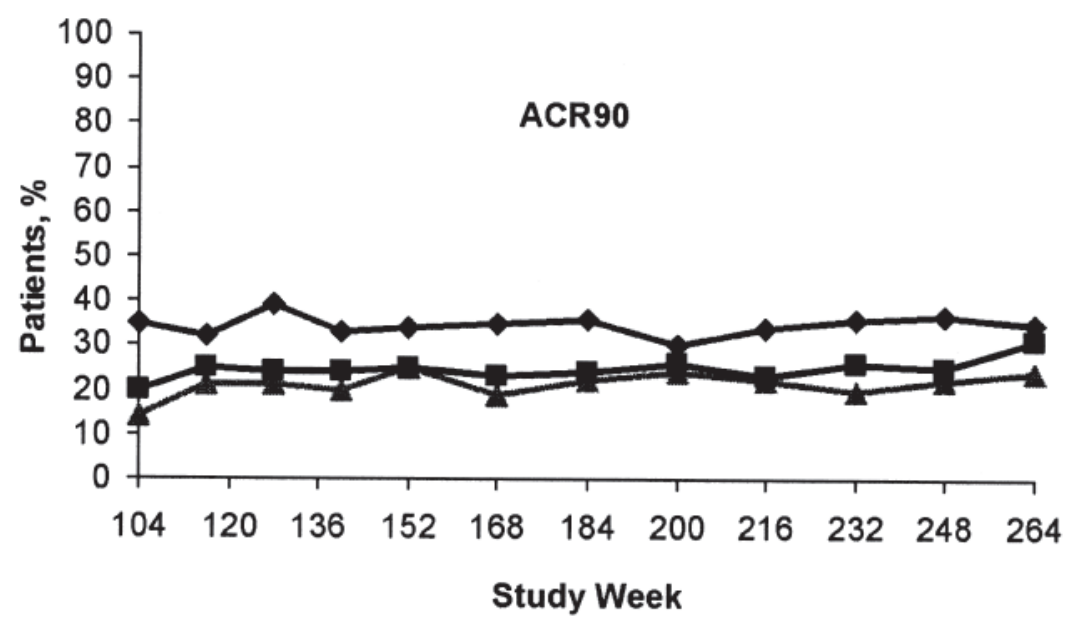

Figure 2. Percentages of patients demonstrating 28-joint Disease Activity Score (DAS28) remission (A), $70 \%$ improvement in the American College of Rheumatology rating scale response criteria (ACR70) (B), and ACR90 (C) during the open-label period. Includes all patients with nonmissing data at each timepoint. 


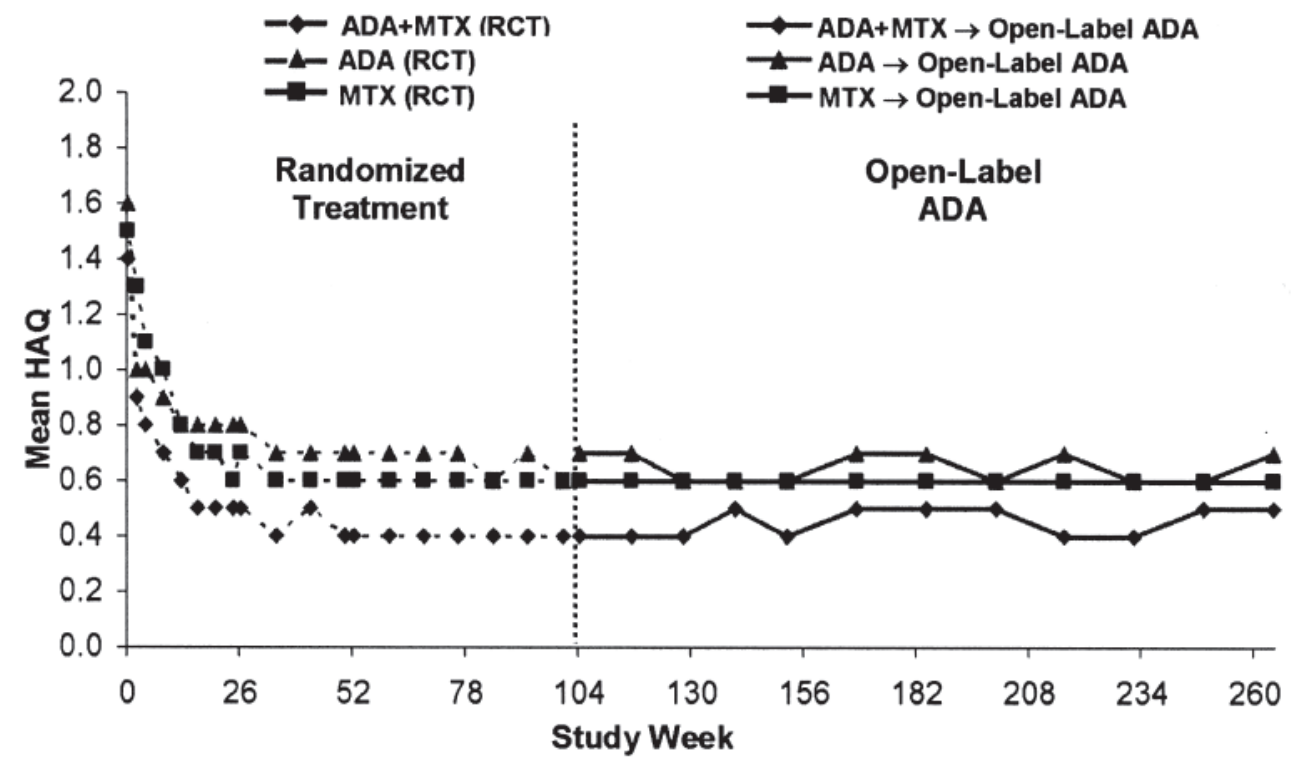

Figure 3. Mean Health Assessment Questionnaire (HAQ) scores during randomized and open-label adalimumab (ADA) treatment. Includes all patients with nonmissing data at each timepoint. MTX: methotrexate; RCT: randomized controlled trial.

\section{DISCUSSION}

Although the benefit of combination therapy with a TNF antagonist plus MTX has been established in early RA, there is a need to understand the longterm outcomes of this therapeutic strategy ${ }^{11,16,17}$. The design of PREMIER has several characteristics that make it unique and important for the evaluation of therapeutic options and longterm outcomes in the treatment of early RA. The study will ultimately follow patients for 10 years to collect both radiographic and clinical measures throughout the extended treatment period for patients with early RA. We were able to evaluate the interim 5-year data by the 3 aspects of disease control in RA therapy: clinical remission, inhibition of radiographic progression, and physical function.

Of the original 799 patients randomized in the 2-year blinded portion of PREMIER, $49 \%$ completed 5 years in the clinical trial ( 2 years of blinded therapy plus 3 years in the open-label extension portion of the trial). In the current 5-year analysis, patients who received initial combination therapy for 2 years experienced better comprehensive disease control over 5 years than patients who received initial therapy with either MTX or ADA alone. The advantage of receiving combination therapy during the first 2 years of treatment was discernible throughout the open-label period, although all 3 treatment groups received the same open-label ADA therapy (with variable MTX use) during Years 3 to 5. The results indicate a window of opportunity to influence the disease course with early, aggressive treatment and suggest that there are consequences associated with delay of combination treatment in patients whose disease activity warrants such therapy.
How to define remission in RA has been a topic of debate $^{18}$. Our operational definition of comprehensive disease remission included a set of criteria addressing each of the treatment goals for RA. At Year 5 of PREMIER, the combination therapy arm had only a minimal advantage over the monotherapy arms on the single measure of DAS28 clinical remission. When normal function was added to DAS28 clinical remission for a combined outcome measure, the difference between the combination group and either monotherapy group was slightly amplified. However, when normal function and/or radiographic nonprogression were also considered, more than twice as many patients had comprehensive disease remission in the combination therapy group than the monotherapy groups. Based on these observations, it appears that marked differences in longterm outcomes, even in early RA, were largely due to measures that identify the cumulative consequences of disease activity over time - radiographic progression and, to a lesser degree, impaired physical function. Given the proven efficacy of biologic therapy in RA, it is no longer sufficient to aim only for clinical remission. The data presented here provide insight into what can now be considered an achievable management goal in the treatment of early RA: clinical remission, radiographic nonprogression, and normal function.

The importance of assessing both clinical and radiographic outcomes when monitoring treatment for RA over long periods cannot be overemphasized. Measures of inflammation and joint damage may be dissociated ${ }^{19}$, and radiographic progression may still occur during periods of low disease activity, as reported with MTX monotherapy ${ }^{20}$.

Personal non-commercial use only. The Journal of Rheumatology Copyright $@$ 2010 . All rights reserved. 
A

$\because A D A+M T X(R C T)$

- - ADA (RCT)

- - MTX (RCT)

ADA+MTX $\rightarrow$ Open-Label ADA ( $=124)$

$A D A \rightarrow$ Open-Label ADA ( $N=115)$

MTX $\rightarrow$ Open-Label ADA ( $N=115)$

mTSS

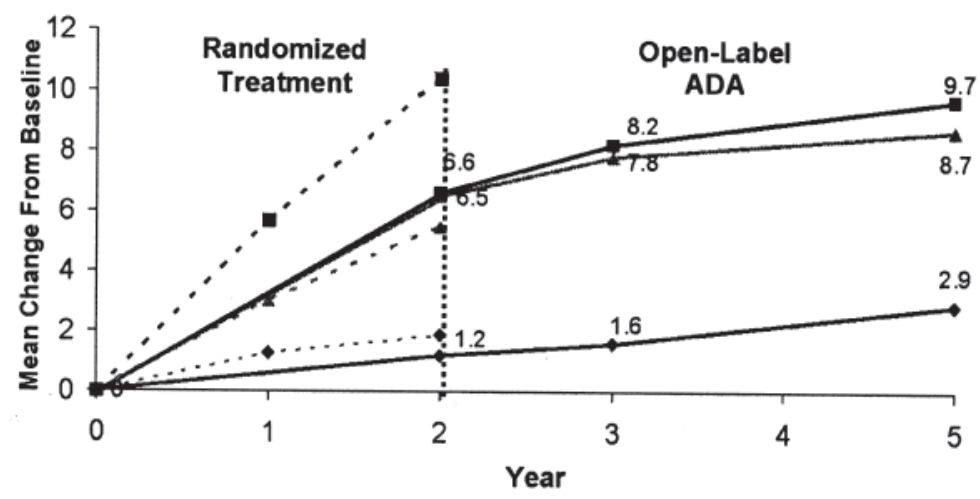

B

\section{JE Score}

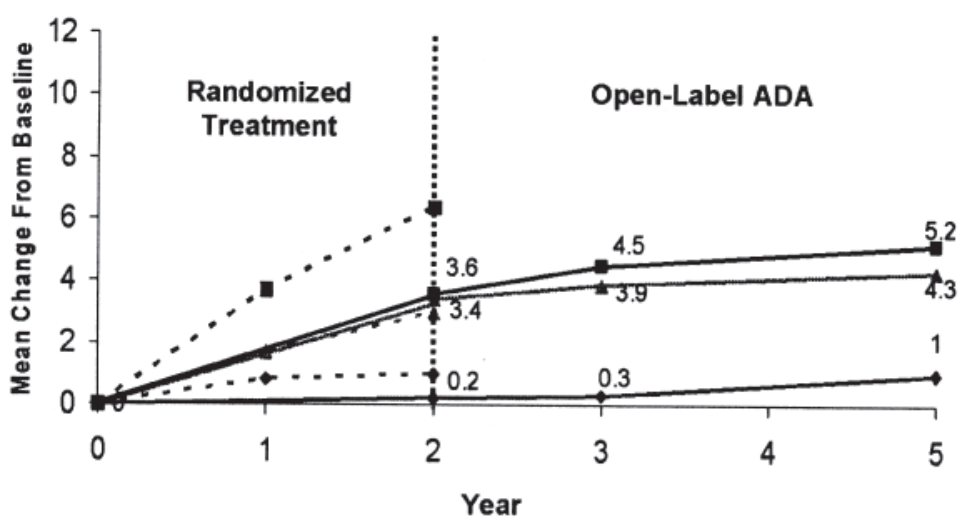

C

JSN Score

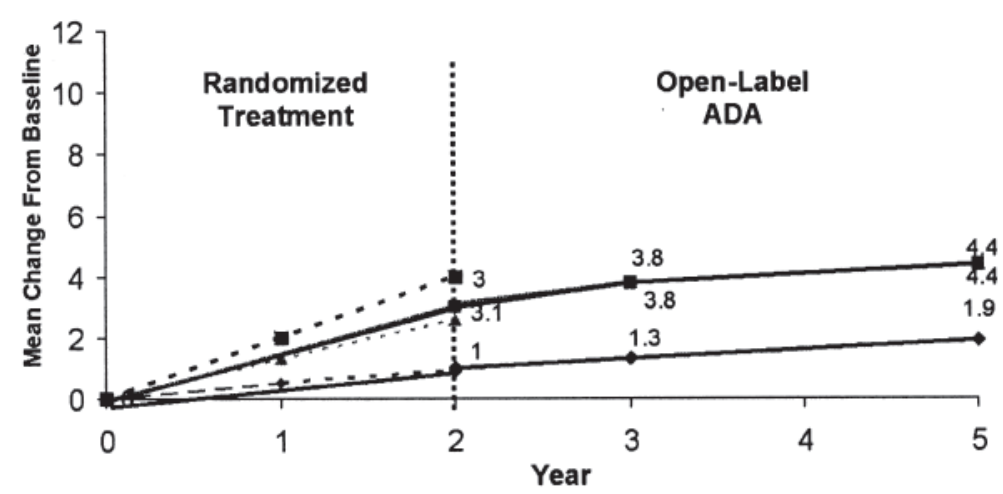

Figure 4. Radiographic scores from baseline to Year 5. Broken lines represent the full randomized study population. Solid lines represent the 5-year completers cohort. A, B, and C present results for modified total Sharp score (mTSS), joint erosion (JE), and joint space narrowing (JSN), respectively. ADA: adalimumab; MTX: methotrexate; RCT: randomized controlled trial. 


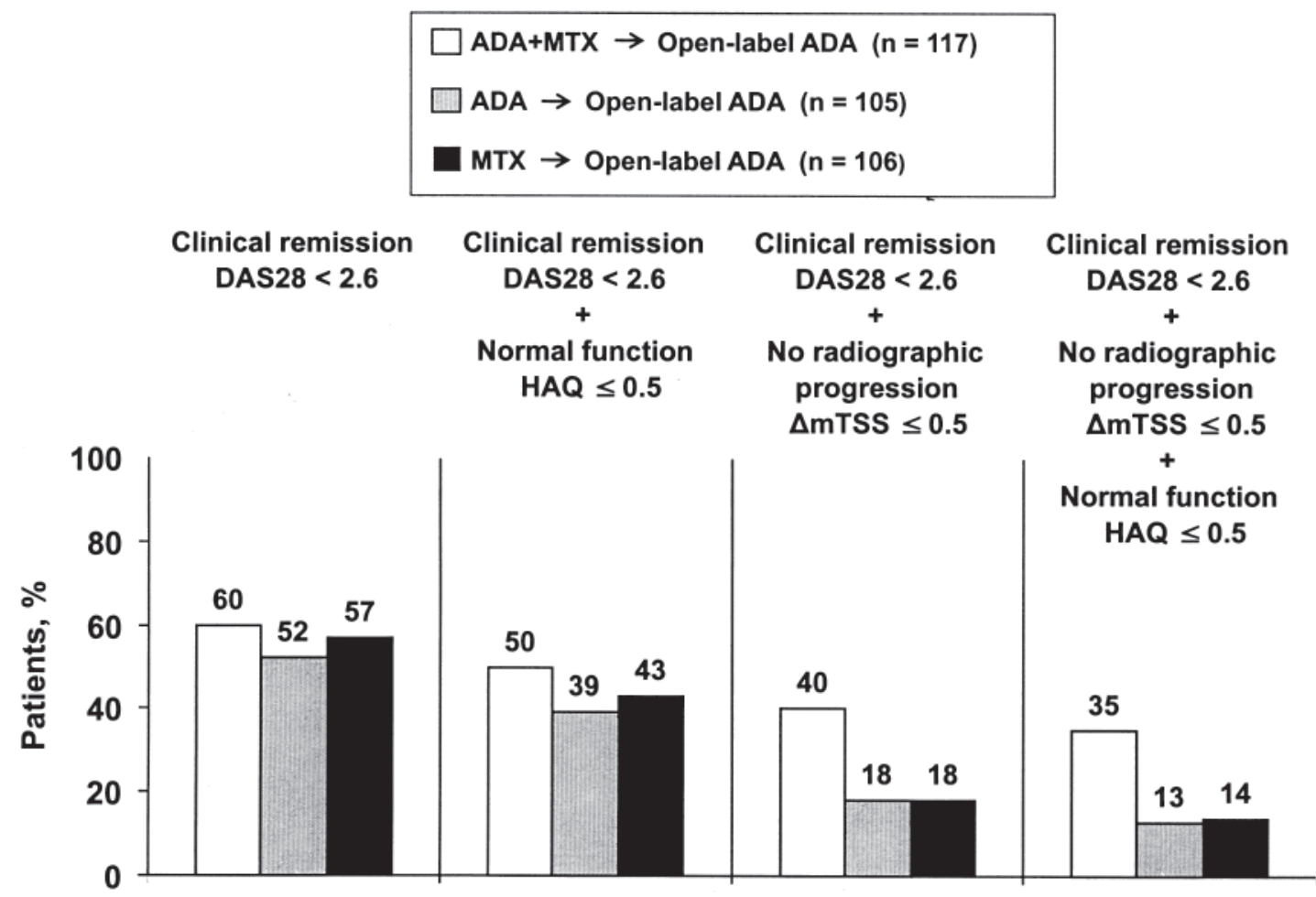

Figure 5. Percentages of patients in the 5-year completers cohort with nonmissing data who met triple criteria for comprehensive disease remission at Year 5. ADA: adalimumab; DAS28: 28-joint Disease Activity Score; HAQ: Health Assessment Questionnaire; mTSS: modified total Sharp score; MTX: methotrexate.

Disease activity measures (e.g., DAS28) and clinical response measures (e.g., ACR response criteria) primarily reflect the current status of the disease in terms of inflammation and the effectiveness of therapy at a specific point in time. These outcome measures do not necessarily identify the consequences of prior therapy on disease progression. On the other hand, structural damage (as captured by radiographic progression) and changes in function (measured by the HAQ) are affected by the history of disease management and control and are more likely to reflect irreversible damage $^{21}$.

During the PREMIER open-label period, MTX could be added to open-label ADA at the investigators' discretion. Almost half of the patients who entered the open-label period received MTX for 12 or more consecutive weeks. The mean DAS28 scores prior to starting MTX in the open-label period were just slightly greater than the cutoff value for low disease activity (3.2) and suggest that either (1) investigators had a lower threshold for initiating combination therapy in patients who had already undergone 2 years of therapy in the double-blind period, some of whom were likely to have had good clinical response when they entered the open-label period; or (2) investigators were making clinical management decisions based on the growing evidence of therapeutic benefit with combination therapy $5,6,7,11,16$. Both scenarios are indicative of the higher expectations physicians have for disease control in RA with the unprecedented efficacy of biologic therapies. However, patients who were treated with concomitant MTX during the open-label period did not have notably better ACR50 responses or DAS28 remission rates in the GEE analysis than those who did not receive MTX during the open-label period. What made a difference in the longterm outcomes at Year 5 was the treatment effect during the initial 2-year, double-blind period of the study.

The efficacy and safety data in this analysis of 5 years of treatment in PREMIER suggest a favorable benefit-risk profile for use of ADA in the early RA population. Overall, rates of SAE during Years 3 to 5 in the open-label period were similar to or numerically lower than those reported previously in ADA RA clinical trials ${ }^{22}$.

There are several limitations of this study. As with all open-label extension studies, there are challenges in presenting these longterm data correctly. On one hand, longterm data are warranted because of the chronicity of the disease. On the other hand, longterm data are hampered by selective dropout, and data are available only for those patients still followed in the study. Therefore, data are based on a group of patients that is smaller than the original randomized population. There is most likely an "enrichment" of patients doing well, as those who remain in the study tend to do better than those who drop out. Nevertheless, data analyses such as this 5-year PREMIER report offer an Personal non-commercial use only. The Journal of Rheumatology Copyright (c) 2010. All rights reserved. 
opportunity to observe the longterm efficacy and safety of treatment in patients who are monitored regularly within the context of a clinical trial. Although the clinical and safety results might be biased toward better results, as the patients who experience clinical benefit remain in the study, the comparison of radiographic data between treatment groups might be less impressive through this approach. For interpretation of the longterm radiographic progression data, we also limited the analysis to the 5-year completers cohort. Hence, Year 5 data were compared with the baseline, Year 2, and Year 3 data from exactly the same patients, instead of being compared with the full intention-to-treat population. This approach might have contributed to narrowing the gap between the 3 treatment groups in terms of radiographic outcomes as patients with worse radiographic progression are no longer in the study.

Another potential limitation of the study is that during open-label ADA treatment, MTX was allowed at the discretion of the investigator. Although this makes the data somewhat more difficult to interpret, the treatment conditions were more similar to actual clinical practice, and results from the GEE repeated-measures logistic model did not point to a relationship between clinical efficacy and MTX use in the open-label period.

In summary, 2 years of combination treatment with ADA plus MTX led to better longterm outcomes than either ADA or MTX monotherapy over 5 years of treatment in the PREMIER trial. More patients in the initial combination treatment group had clinical remission, inhibition of structural damage, and normal function than in either monotherapy group at Year 5. The longterm efficacy and safety data suggest a favorable benefit-risk profile for the use of ADA over 5 years in patients with early RA.

\section{ACKNOWLEDGMENT}

The authors thank Ellen Stoltzfus, PhD, of JK Associates, Inc., and Michael A. Nissen, ELS, of Abbott Laboratories, for their editorial support in the development and revision of this report.

\section{REFERENCES}

1. van der Heijde DM. Joint erosions and patients with early rheumatoid arthritis. Br J Rheumatol 1995;34 Suppl 2:74-8.

2. Lindqvist E, Jonsson K, Saxne T, Eberhardt K. Course of radiographic damage over 10 years in a cohort with early rheumatoid arthritis. Ann Rheum Dis 2003;62:611-6.

3. Emery P, Breedveld FC, Dougados M, Kalden JR, Schiff MH, Smolen JS. Early referral recommendation for newly diagnosed rheumatoid arthritis: evidence based development of a clinical guide. Ann Rheum Dis 2002;61:290-7.

4. Jamal S, Patra K, Keystone EC. Response to adalimumab in patients with early versus established rheumatoid arthritis: DE019 randomized controlled trial subanalysis. Clin Rheumatol 2009;28:413-9.

5. Keystone EC, Kavanaugh AF, Sharp JT, Tannenbaum H, Hua Y, Teoh LS, et al. Radiographic, clinical, and functional outcomes of treatment with adalimumab (a human anti-tumor necrosis factor monoclonal antibody) in patients with active rheumatoid arthritis receiving concomitant methotrexate therapy. Arthritis Rheum 2004;50:1400-11.

6. Klareskog L, van der Heijde D, de Jager JP, Gough A, Kalden J, Malaise M, et al. Therapeutic effect of the combination of etanercept and methotrexate compared with each treatment alone in patients with rheumatoid arthritis: double-blind randomised controlled trial. Lancet 2004;363:675-81.

7. Lipsky PE, van der Heijde DMFM, St. Clair EW, Furst DE, Breedveld FC, Kalden JR, et al. Infliximab and methotrexate in the treatment of rheumatoid arthritis. N Engl J Med 2000; 343:1594-602.

8. Strand V, Singh JA. Improved health-related quality of life with effective disease-modifying antirheumatic drugs: evidence from randomized controlled trials. Am J Manag Care 2008;14:234-54.

9. Bejarano V, Reece R, Quinn M, Conaghan PG, Keenan A-M, Walker D, et al. Job loss in patients with early rheumatoid arthritis can be prevented by early use of the anti-tumor necrosis factor adalimumab: results of the PRevention Of Work Disability (PROWD) study. Arthritis Care Res 2008;59:1467-74.

10. van Vollenhoven RF, Cifaldi MA, Ray S, Chen N, Weisman MH. Improvement in workplace and household productivity for patients with early rheumatoid arthritis (RA) treated with adalimumab plus methotrexate: work outcomes and their correlations with clinical and radiographic measures from a randomized controlled trial companion study. Arthritis Care Res 2010;62:226-34.

11. Breedveld FC, Weisman MH, Kavanaugh AF, Cohen SB, Pavelka $\mathrm{K}$, van Vollenhoven R, et al. The PREMIER study: a multicenter, randomized, double-blind clinical trial of combination therapy with adalimumab plus methotrexate vs. methotrexate alone or adalimumab alone in patients with early, aggressive rheumatoid arthritis who had not had previous methotrexate treatment. Arthritis Rheum 2006;54:26-37.

12. Arnett FC, Edworthy SM, Bloch DA, McShane DJ, Fries JF, Cooper NS, et al. The American Rheumatism Association 1987 revised criteria for the classification of rheumatoid arthritis. Arthritis Rheum 1988;31:315-24.

13. Prevoo ML, van 't Hof MA, Kuper HH, van Leeuwen MA, van de Putte LB, van Riel PL. Modified disease activity scores that include twenty-eight-joint counts. Development and validation in a prospective longitudinal study of patients with rheumatoid arthritis. Arthritis Rheum 1995;38:44-8.

14. Fries JF, Spitz P, Kraines RG, Holman HR. Measurement of patient outcome in arthritis. Arthritis Rheum 1980;23:137-45.

15. American College of Rheumatology Subcommittee on Rheumatoid Arthritis Guidelines. Guidelines for the management of rheumatoid arthritis: 2002 update. Arthritis Rheum 2002;46:328-46.

16. St. Clair EW, van der Heijde DM, Smolen JS, Maini RN, Bathon JM, Emery P, et al. Combination of infliximab and methotrexate therapy for early rheumatoid arthritis: a randomized, controlled trial. Arthritis Rheum 2004;50:3432-43.

17. Emery P, Breedveld F, Hall S, Durez P, Chang D, Robertson D, et al. Comparison of methotrexate monotherapy with a combination of methotrexate and etanercept in active, early, moderate to severe rheumatoid arthritis (COMET): a randomised, double-blind, parallel treatment trial. Lancet 2008;372:375-82.

18. Pincus T, Kavanaugh A, Aletaha D, Smolen J. Complexities in defining remission in rheumatic diseases. Clin Exp Rheumatol 2006;24:S1-6.

19. Smolen JS, Han C, Bala M, Maini RN, Kalden JR, van der Heijde D, et al. ATTRACT Study Group. Evidence of radiographic benefit of treatment with infliximab plus methotrexate in rheumatoid arthritis patients who had no clinical improvement: a detailed subanalysis of data from the anti-tumor necrosis factor trial in rheumatoid arthritis with concomitant therapy study. Arthritis Rheum 2005;52:1020-30. 
20. Smolen JS, Han C, van der Heijde DM, Emery P, Bathon JM, Keystone E, et al. Active-Controlled Study of Patients Receiving Infliximab for the Treatment of Rheumatoid Arthritis of Early Onset (ASPIRE) Study Group. Radiographic changes in rheumatoid arthritis patients attaining different disease activity states with methotrexate monotherapy and infliximab plus methotrexate: the impacts of remission and tumour necrosis factor blockade. Ann Rheum Dis 2009;68:823-7.
21. Aletaha D, Smolen J, Ward MM. Measuring function in rheumatoid arthritis: identifying reversible and irreversible components. Arthritis Rheum 2006;54:2784-92.

22. Burmester GR, Mease P, Dijkmans BAC, Gordon K, Lovell D, Panaccione R, et al. Adalimumab safety and mortality rates from global clinical trials of six immune-mediated inflammatory diseases. Ann Rheum Dis 2009;68:1863-9. 